\title{
The European Working Time Directive:
} less means less

The European Working Time Directive (EWTD) was introduced in the UK in $1998 .{ }^{1}$ The legislation supports proposals made 5 years earlier in the European Union as part of the Social Chapter of the Maastricht Treaty to protect workers from being compelled to work excessive hours. Even at this early stage the potential implications for medical training and practice were recognised. ${ }^{2}$ Doctors in training were seen as a special group and the Directive acknowledged the need to protect continuity in a number of settings. However, there is now increasing evidence that the Directive is having serious adverse effects on both medical training and patient care. Described by an anonymous senior manager as 'the worst legislation ever inflicted on the NHS', the Directive limits hours of work to an average of 48 each week. Why 48? We do not know how this figure was plucked out of the air, but the legislation was clearly not designed for the professions, particularly those with 24hour responsibility for sick patients. Hospitals in the rest of Europe either ignore the Directive, or use the opportunities for voluntary opt-out. The present UK government, with its tradition of 'goldplating' European law, and our centralist and bureaucratic healthcare system have tried to implement it to the letter.

Over the last 10 years, hours worked by junior hospital doctors have been driven down, and on paper most hospitals now have 48-hour compliant rotas. Where there used to be a team of doctors on call there is now usually a single person. In the course of the day there will be multiple handovers as part of the implementation of shift working, and each handover is an accident waiting to happen. Continuity of care has deteriorated significantly, provided by very stretched consultants as best they can. The 'firm' structure, so important for bonding and apprenticeship training, has largely gone. Inevitably the scarce junior doctor time available is being used for out-of-hours care at the expense of daytime training. Surgeons in particular are affected badly. Many of their elective clinics and operating lists where they learn their trade have been removed from schedules. Even when on duty at night they are not gaining proper experience of emergency care. A recent National Confidential Enquiry into Patient Outcome and Death report ${ }^{3}$ pointed out that more and more emergency surgery is being done by consultants operating alone, as the single, very busy trainee on duty does his or her best to cope with sick patients on the wards and cannot get to the operating theatre.

And, paradoxically, the health of junior medical staff appears to have been adversely affected. A recent study from one NHS trust ${ }^{4}$ has found a notable increase in episodes of sick leave among junior doctors since the introduction of an EWTD-compliant rota, although the same study did not identify any negative impacts on patient care.

These are problems for trainees in other branches of medicine. The ability to assess and identify serious illness is a core skill of medicine and a crucial part of general practice. As trainees have less and less direct contact with sick patients, the development of their clinical skills is correspondingly impaired. Taken with the much reduced involvement in out-of-hours care by GPs at all career stages, this creates potentially unsafe structures for both training and practice.

A further problem relates to teaching undergraduate medical students. Consultants are now so busy trying to provide some sort of continuity of care to patients and fire-fighting the problems resulting from thin layers of medical cover with multiple handovers, that they are missing their teaching sessions. Junior doctors, from whom as students we all learnt so much, both in formal teaching and informally by day and by night, no longer have time to teach as they are sent off on unwanted rest days. When on call, the previous team which had time and space to show patients to the students has been replaced by a single doctor, worked off his or her feet, with not enough time to deal with sick patients, never mind show the medical student what is going on. This is also an issue for trainee development, as teaching students and more junior medical colleagues has always been an integral part of training.

In our big medical schools with very large undergraduate numbers, students' hands-on clinical experiences are reduced. Shorter hospital stays mean that patients are less available for clinical teaching, necessitating greater reliance on simulations and virtual learning environments. If curricular changes lead to further reductions in the amount of factual knowledge acquired by students, the situation may be even starker. How will young doctors acquire the necessary knowledge base if they have not learnt it at medical school or have experience of the multiple diseases they must know about? The result of all this, unless something is done quickly, will be a generation of doctors who have not had sufficient experience to make a diagnosis in a sick patient, and to make decisions about management, quite apart from being able to carry out procedures which require skill and practice.

Solutions put forward include the reconfiguration of hospitals and substantial increases in the numbers of doctors, but it is unclear whether these will happen or, indeed, are desirable in the wider context of the NHS. Improved training methods and curricula will help, but will do nothing for the deficiency in hand-on experience. Any administration needs to examine ways of avoiding the restrictions of the EWTD and restoring junior doctors' hours to levels consistent with proper training and a safer service.

\section{John Black,}

President, Royal College of Surgeons of England, London.

\section{Roger Jones,} Editor, BJGP. 


\section{Provenance}

Commissioned; not peer reviewed.

\section{REFERENCES}

1. Department of Health. What is the European Working Time Directive?

http://www.dh.gov.uk/en/Managingyourorganisation/W orkforce/Workforceplanninganddevelopment/European workingtimedirective/DH_077304 (accessed 8 Apr 2010).

2. Royal College of Physicians. European Working Time Directive (EWTD). Why is the EWTD relevant to me? http://www.rcplondon.ac.uk/professional- issues/workforce/workforce-issues/pages/ewtd.aspx (accessed 8 Apr 2010).

3. National Confidential Enquiry into Patient Outcome and Death. Caring to the End? A review of the care of patients who died in hospital within four days of admission. http://www.ncepod.org.uk/2009dah.htm (accessed 8 Apr 2010).

4. McIntyre HF, Winfield S, Te HS, Crook D Implementation of the European Working Time Directive in an NHS trust: impact on patient care and junior doctor welfare. Clin Med 2010; 10(2): 134-137.

\section{ADDRESS FOR CORRESPONDENCE}

\section{John Black}

The Royal College of Surgeons of England, 35-43 Lincoln's Inn Fields, London WC2A 3PE.

Email: john.black@rcseng.ac.uk

\section{Bipolar spectrum disorders in primary care: optimising diagnosis and treatment}

Depression is an extremely common presentation in primary care and the public health importance of depressive disorders are now very well established. ${ }^{1,2}$ Patients with bipolar affective disorder (who experience episodes of depression alternating with episodes of mania or hypomania), frequently present to their GPs with difficult-to-treat depressive episodes. Indeed, for most of these patients, depressive symptoms (rather than manic symptoms) dominate the long-term clinical course of their illness.

Bipolar disorder type I (BD-I; depression alternating with mania) affects around $1 \%$ of the population and bipolar disorder type II (BD-II; depression alternating with hypomania) affects a further $2-3 \% .^{3}$ Although the clinical features of BD-I and BD-II are widely known, it is less well recognised that the boundary between bipolar disorder and recurrent unipolar depression is far from clear-cut. ${ }^{4}$ A significant proportion of patients with unipolar depression experience mild or brief episodes of hypomania which fall below the threshold for a formal diagnosis of BD-I or BD-II. ${ }^{5-7}$
These 'bipolar spectrum' patients often have patterns of depressive episodes, comorbidities, and treatment responses that differ from those with more straightforward unipolar depression and which, therefore, require a different approach to diagnosis and management. This generally under-recognised issue has far-reaching implications for the way in which clinicians, particularly GPs, approach the assessment and management of all of their depressed patients.

Within the research community, there is an emerging consensus that the diagnostic criteria for hypomania are overly restrictive and result in many patients with significant bipolar symptoms being placed within the broad diagnostic category of unipolar depression. ${ }^{7-9}$ Converging evidence from a number of studies from around the world suggests that at least $25 \%$ of patients with recurrent unipolar depression may be better classified as having a broadlydefined bipolar spectrum disorder. ${ }^{5-7,10-12}$ Importantly, these patients have similar rates of bipolar family history and comparable levels of health service use, long-term illness severity, and psychosocial morbidity as patients with bipolar disorder diagnosed according to the formal DSM-IV criteria. . $, 7,10^{\circ}$ Furthermore, certain clinical subgroups of depressed patients, such as those with an especially severe or early-onset of depression ${ }^{13,14}$ or those with treatment resistance to antidepressants, ${ }^{15}$ appear to have particularly high rates of unrecognised bipolar disorder.

For a number of reasons, BD-I and BDII disorder (and, by extension, bipolar spectrum disorder) can be very difficult to diagnose. ${ }^{16}$ Most BD-I and BD-II patients will experience a delay of several years between their first onset of significant manic symptoms and receiving the correct diagnosis, and misdiagnosis in the interim as unipolar depression, personality disorder, a primary drug or alcohol problem, or even schizophrenia is relatively common. ${ }^{16-19}$ Given that depression (rather than mania) dominates the natural history of bipolar disorder, patients are much more likely to seek help, usually from their GP, during their 